\title{
Adaptive unscented Kalman filter for parameter and state estimation of nonlinear high-speed objects
}

\author{
Fang Deng ${ }^{1,2}$, Jie Chen ${ }^{1,2}$, and Chen Chen ${ }^{1,2, *}$ \\ 1. School of Automation, Beijing Institute of Technology, Beijing 100081, China; \\ 2. Key Laboratory of Intelligent Control and Decision of Complex Systems, Beijing 100081, China
}

\begin{abstract}
An adaptive unscented Kalman filter (AUKF) and an augmented state method are employed to estimate the timevarying parameters and states of a kind of nonlinear high-speed objects. A strong tracking filter is employed to improve the tracking ability and robustness of unscented Kalman filter (UKF) when the process noise is inaccuracy, and wavelet transform is used to improve the estimate accuracy by the variance of measurement noise. An augmented square-root framework is utilized to improve the numerical stability and accuracy of UKF. Monte Carlo simulations and applications in the rapid trajectory estimation of hypersonic artillery shells confirm the effectiveness of the proposed method.
\end{abstract}

Keywords: parameter estimation, state estimation, unscented Kalman filter (UKF), strong tracking filter, wavelet transform.

DOI: 10.1109/JSEE.2013.00076

\section{Introduction}

Parameter and state estimation plays a fundamental role in the design and tracking of missiles, aircraft and shells, as well as in a number of related applications, such as simulation of objects dynamics and fault detection and diagnosis. The parameter and state estimation has attracted great attention in the field of aircraft and its ballistic researches.

When we estimate parameters and states of missiles, artillery shells and other nonlinear high-speed objects, we need to face the following problems. First, these systems always involve a strong nonlinearity. Second, they always have inaccurate measurement noises, which are either Gaussian or non-Gaussian. Third, the process noise is generally imprecise. Considering the characteristics mentioned above, a nonlinear parameter and state estimation method, which is precise, fast, robust and adaptive, was proposed.

Manuscript received April 10, 2012.

*Corresponding author.

This work was supported by the National Natural Science Foundation of China (61304254), the National Science Foundation for Distinguished Young Scholars of China (60925011), and the Provincial and Ministerial Key Fund of China (9140A07010511BQ0105).
The extended Kalman filter (EKF) $[1,2]$ has undoubtedly been a dominating technique for the nonlinear state estimation. Nevertheless, due to the stronger nonlinearity between the range rate and the target state, the truncation error is too large to be omitted, and the poor performance of EKF is undoubtable. The covariance matching technique attempts to make the filter residuals consistent with their theoretical covariances, such as the strong tracking Kalman filter (STKF) [3-5]. Moreover, the derivation of the Jacobian matrix, necessary for the first order approximation, is important in many applications. Several available estimators, such as re-iteration, high-order filters and statistical linearization [6], are more complex than EKF and STKF. These modifications generally improve the estimation accuracy with more complexity of computation and implementation. Particle filtering [7] (PF) is a Monte Carlo simulation based nonlinear filtering algorithm. The main problem with $\mathrm{PF}$ is the degradation of particles [8] and its computation complexity in real applications in the parameters and states estimation of high-speed objects.

The unscented Kalman filter (UKF) [9-11], proposed by Julier and Uhlman, theoretically improves EKF for the state and parameter estimation. The linearization of the UKF is avoided by an unscented transformation, and at least the second order accuracy is provided. Furthermore, it is not necessary to compute explicitly the Jacobians or Hessians in the UKF which avoids the particle recession of PF due to adopting the deterministic sampling strategy. The UKF can solve the strongly nonlinear problems with satisfactory robustness and stability properties. Therefore, the UKF is a nonlinear filter with high precision, strong stability and small computational burden, which is suitable for estimating parameters and states of high-speed objects. In recent years, the UKF has been widely used in estimation of states and parameters [12-14], control [15], target tracking [16,17], navigation [18,19], map building $[20,21]$ and fault diagnosis [22]. At the same time, many scholars have tried to improve the UKF in terms of its nu- 
merical stability and computational speed, e.g., a decrease sigma points UKF [23] and square root decomposition of the UKF $[24,25]$.

In this paper, we try to improve the performance of general UKF in the following aspects, ability to estimate the measurement noise estimation, robustness in imprecise process noise, accuracy, tracking ability, and numerical stability. The adaptive UKF (AUKF) is adopted in the rapid trajectory estimation of nonlinear high-speed objects. For the estimation of catastrophe parameters and states, a strong tracking filter is employed, and the wavelet transform is utilized to estimate the variance of measurement noise to improve the estimation accuracy and robustness. We use a square-root augmented algorithm to improve the numerical stability and accuracy of AUKF. Theoretical analysis and Monte Carlo simulation demonstrate that the accuracy and effectiveness of AUKF is better than the strong tracking EKF and the normal UKF.

This paper is organized as follows: Section 2 presents the features of nonlinear high-speed objects and the estimation of states and time-varying parameters of highspeed objects. Section 3 presents an improved adaptive UKF. Section 4 gives a numerical example. Applications in the artillery shell are given in Section 5, and conclusions are drawn in Section 6.

\section{Estimation of states and time-varying parameters of nonlinear high-speed objects}

\subsection{Mathematical description of nonlinear high-speed objects}

Consider the following nonlinear high-speed objects with

$$
\begin{gathered}
\boldsymbol{x}_{k+1}=\boldsymbol{f}\left(\boldsymbol{x}_{k}, \boldsymbol{\theta}_{k}\right)+\boldsymbol{v}_{k} \\
\boldsymbol{y}_{k+1}=\boldsymbol{h}\left(\boldsymbol{x}_{k+1}, \boldsymbol{\theta}_{k+1}\right)+\boldsymbol{w}_{k+1}
\end{gathered}
$$

where $\boldsymbol{x}_{k+1} \in \mathbf{R}^{n}$ is a vector of the state variable, $\boldsymbol{v}_{k} \in$ $\mathbf{R}^{n}$ is a vector of the plant noise, the function $\boldsymbol{f}$ is a differentiable function of the state vector $\boldsymbol{x}_{k}, \boldsymbol{y}_{k+1} \in \mathbf{R}^{m}$ is a vector of the measured variable, the function $\boldsymbol{h}$ is a differentiable function of the state vector $\boldsymbol{x}_{k+1}, \boldsymbol{w}_{k+1} \in \mathbf{R}^{m}$ is a vector of the measurement noise, and $\boldsymbol{\theta}_{k}$ is a $p$-dimensional time-varying parameter vector to be identified.

It is assumed that the additive noise vectors, $\boldsymbol{v}_{k}$ and $\boldsymbol{w}_{k+1}$, are Gaussian uncorrelated white noises. $\mathrm{E}\left(\boldsymbol{v}_{k}\right)=$ $\mathrm{E}\left(\boldsymbol{w}_{k+1}\right)=0$, and their covariances are $\boldsymbol{Q}_{k}$ and $\boldsymbol{R}_{k+1}$ respectively. The characteristics of nonlinear high-speed objects mentioned in this paper, e.g., missiles, aircraft and shells, are as follows:

(i) The velocity of these systems is faster than Mach one and the change of velocity is very big, e.g., the max velocity of the artillery shell is Mach 3 and the missile is about Mach 10. The acceleration of the artillery shell is about thousands of gravitational acceleration and the missile is about 10 gravitational acceleration.

(ii) When the high-speed objects fly in the air, friction and resistance of air can produce complex dynamic behaviors, such as vibration and swing. There is a strong nonlinearity in the movement of the high-speed objects. Therefore, it is difficult to model and control the high-speed objects.

(iii) High-speed objects have dynamic stability. Some high-speed objects have to rely on strong rotation to keep the stability, e.g., artillery shells, and rotation also brings us a very complex expression of the movement.

(iv) These systems are strong nonlinear systems. There are fast varying or mutation and nonlinear parameters and states. Some parameters of the systems are nonlinear piecewise functions and there is a strong coupling among parameters and states.

(v) The observation data have a time-varying covariance, i.e., $\boldsymbol{R}_{k+1}$ is not a fixed value, and the process noise is imprecise.

(vi) The systems are always described by high dimensional nonanalytic differential equations. The differential equations do not have exact analytical solutions but numerically approximate solutions.

\subsection{Estimation of states and time-varying parameters}

For estimating the parameters, the state variable $\boldsymbol{x}_{k}$ and the nonlinear parameter $\theta_{k}$ constitute the augmented state vector,

$$
\begin{gathered}
\boldsymbol{X}_{k+1}=\left[\begin{array}{c}
\boldsymbol{x}_{k+1} \\
\boldsymbol{\theta}_{k+1}
\end{array}\right]=\left[\begin{array}{c}
\boldsymbol{f}\left(\boldsymbol{x}_{k}, \boldsymbol{\theta}_{k}\right)+\boldsymbol{v}_{k} \\
\boldsymbol{\theta}_{k}+\boldsymbol{w}_{k}
\end{array}\right]= \\
\boldsymbol{f}\left(\boldsymbol{X}_{k+1}\right)+\boldsymbol{V}_{k}
\end{gathered}
$$

where $\boldsymbol{X}_{k}=\left[\boldsymbol{x}_{k}, \boldsymbol{\theta}_{k}\right]^{\mathrm{T}}, \boldsymbol{V}_{k}=\left[\boldsymbol{v}_{k}, \boldsymbol{w}_{k}\right]^{\mathrm{T}}, \boldsymbol{w}_{k}$ is a zeromean white noise with variance $\boldsymbol{W}_{k}$, so the noise covariance matrix of the augmented state equation is

$$
\boldsymbol{Q}_{k}^{a}=\left[\begin{array}{cc}
\boldsymbol{Q}_{k} & 0 \\
0 & \boldsymbol{W}_{k}
\end{array}\right] .
$$

After the state expansion, a nonlinear filter can be utilized to estimate the parameters and states of the system. An adaptive UKF algorithm is utilized to estimate the parameters and states in the next section.

\section{Adaptive UKF}

\subsection{Standard UKF}

Considering the nonlinear high-speed objects from (1) to (2), the standard UKF $[9,10]$ equations are given as follows.

3.1.1 Compution of the sigma points and weights

The $n$-dimensional random variable $\boldsymbol{x}_{k}$ with mean $\hat{\boldsymbol{x}}_{k}$ and covariance $\boldsymbol{P}_{k}$ is approximated by $2 n+1$ weighted samples 
or sigma points selected by the algorithm,

$$
\begin{gathered}
\chi_{k}=\left[\hat{\boldsymbol{x}}_{k}, \hat{\boldsymbol{x}}_{k}+\left(\sqrt{\varpi \boldsymbol{P}_{k}}\right)_{i}, \hat{\boldsymbol{x}}_{k}-\left(\sqrt{\varpi \boldsymbol{P}_{k}}\right)_{i}\right] \\
W_{0}^{(m)}=\eta / \varpi \\
W_{0}^{(c)}=\eta / \varpi+\left(1-\alpha^{2}+\beta\right) \\
W_{i}^{(m)}=W_{i}^{(c)}=1 /(2 n+2 \eta), \quad i=1, \ldots, 2 n \\
\eta=\alpha^{2}(n+\zeta)-n \\
\varpi=n+\eta
\end{gathered}
$$

where $\left(\sqrt{\boldsymbol{P}_{k}}\right)_{i}$ is the $i$ th row or column of the matrix square root of $\boldsymbol{P}_{k}, W_{i}$ is the weight associated with the $i$ th point, and $\eta$ is a scaling parameter. The parameter $\alpha$ determining the spread of the sigma points around $\hat{\boldsymbol{x}}_{k}$ is usually set to a small positive value (e.g. 0.001 ). $\zeta$ is a secondary scaling parameter usually set to 0 , and $\beta$ is utilized to incorporate prior knowledge of the distribution of $\boldsymbol{x}_{k}$ (for Gaussian distributions, $\beta=2$ is optimal).

\subsubsection{State prediction}

Sigma points will be substituted into the nonlinear state equation and observation equation, and the transformation sigma points are evaluated for each of the $0-2 n$ points by

$$
\chi_{k+1 \mid k}=\boldsymbol{f}\left(\chi_{k}\right) .
$$

The mean of the transformed set of sigma points $\hat{\boldsymbol{x}}_{k+1 \mid k}$ is computed by

$$
\hat{\boldsymbol{x}}_{k+1 \mid k}=\sum_{i=0}^{2 n} W_{i}^{(m)} \chi_{i, k+1 \mid k} .
$$

The predicted observation is

$$
\begin{gathered}
\gamma_{k+1 \mid k}=\boldsymbol{h}\left(\boldsymbol{\chi}_{k+1 \mid k}\right) \\
\hat{\boldsymbol{y}}_{k+1 \mid k}=\sum_{i=0}^{2 n} W_{i}^{(m)} \boldsymbol{\gamma}_{i, k+1 \mid k}
\end{gathered}
$$

and the predicted covariance is

$$
\begin{gathered}
\boldsymbol{P}_{k+1 \mid k}=\sum_{i=0}^{2 n} W_{i}^{(c)}\left[\boldsymbol{\chi}_{i, k+1 \mid k}-\hat{\boldsymbol{x}}_{k+1 \mid k}\right] \\
{\left[\boldsymbol{\chi}_{i, k+1 \mid k}-\hat{\boldsymbol{x}}_{k+1 \mid k}\right]^{\mathrm{T}}+\boldsymbol{Q}}
\end{gathered}
$$

where $\chi_{i, k+1 \mid k}, \gamma_{i, k+1 \mid k}$ are the $i$ th row of $\chi_{k+1 \mid k}$ and $\gamma_{k+1 \mid k}$, respectively.

\subsubsection{Measurement update}

Compute the covariance and the cross correlation matrix by

$$
\begin{gathered}
\boldsymbol{P}_{x_{k+1} y_{k+1}}=\sum_{i=0}^{2 n} W_{i}^{(c)}\left[\boldsymbol{\chi}_{i, k+1 \mid k}-\hat{\boldsymbol{x}}_{k+1 \mid k}\right] \\
{\left[\boldsymbol{\gamma}_{i, k+1 \mid k}-\hat{\boldsymbol{y}}_{k+1 \mid k}\right]^{\mathrm{T}}}
\end{gathered}
$$

$$
\begin{gathered}
\boldsymbol{P}_{y_{k+1} y_{k+1}}=\sum_{i=0}^{2 n} W_{i}^{(c)}\left[\boldsymbol{\gamma}_{i, k+1 \mid k}-\hat{\boldsymbol{y}}_{k+1 \mid k}\right] \\
{\left[\boldsymbol{\gamma}_{i, k+1 \mid k}-\hat{\boldsymbol{y}}_{k+1 \mid k}\right]^{\mathrm{T}}+\boldsymbol{R}_{k+1}} \\
\boldsymbol{K}_{k+1}=\boldsymbol{P}_{x_{k+1} y_{k+1}} \boldsymbol{P}_{y_{k+1} y_{k+1}}^{-1}
\end{gathered}
$$

Then update the mean and the covariance

$$
\begin{aligned}
& \boldsymbol{P}_{k+1}=\boldsymbol{P}_{k+1 \mid k}-\boldsymbol{K}_{k+1} \boldsymbol{P}_{y_{k+1} y_{k+1}} \boldsymbol{K}_{k+1}^{\mathrm{T}} \\
& \hat{\boldsymbol{x}}_{k+1}=\hat{\boldsymbol{x}}_{k+1 \mid k}+\boldsymbol{K}_{k+1}\left(\boldsymbol{y}_{k+1}-\hat{\boldsymbol{y}}_{k+1 \mid k}\right) .
\end{aligned}
$$

The standard UKF has an excellent performance in the estimation of general nonlinear systems, but it has a poor performance in tracking mutation parameters and states. When the process noise is imprecise or the measurement noise is time-varying, the accuracy of general UKF would be greatly reduced. Thus, we should improve its ability to track catastrophe parameters and states. The adaptive UKF should also be adapted to the time-varying observation noise and the inaccurate process noise. Furthermore, the adaptive UKF should be stable enough for high-dimension systems.

\subsection{Improvement in tracking ability and robustness against inaccurate process noise}

Although the accuracy of the general UKF is higher than that of EKF, compared with the strong tracking EKF [3], it lacks tracking ability and robustness when the process noise is inaccurate, particularly in the tracking of catastrophe parameters and states. A strong tracking UKF is given in this sub-section to improve the tracking ability and robustness.

Lemma $1[3,26]$ Orthogonal principle: The sufficient condition for a filter called a strong tracking filter is only when the time-varying filter gain matrix is selected online such that the state estimation mean-square error is minimized and the innovations remain orthogonal:

$$
\begin{gathered}
\mathrm{E}\left[\boldsymbol{x}_{k+1}-\hat{\boldsymbol{x}}_{k+1 \mid k+1}\right]\left[\boldsymbol{x}_{k+1}-\hat{\boldsymbol{x}}_{k+1 \mid k+1}\right]^{\mathrm{T}}=\min \\
\mathrm{E}\left[\boldsymbol{\gamma}_{k+1+j} \boldsymbol{\gamma}_{k+1}^{\mathrm{T}}\right]=0, \quad k=0,1, \ldots, n ; j=1,2, \ldots, m .
\end{gathered}
$$

The general UKF uses unscented transform for time updates, with the minimum variance estimation for the measurement update, so it satisfies (21) of the orthogonal principle. Equation (22) essentially requires the residuals at different time steps to keep mutually orthogonal. From Lemma 1, an inference is as follows:

Inference 1 Define $\varepsilon_{k} \triangleq \boldsymbol{x}_{k}-\hat{\boldsymbol{x}}_{k}$, when the strong tracking filter can accurately estimate the state of the system, if $O\left[\left|\varepsilon_{k}\right|^{2}\right] \ll O\left[\left|\varepsilon_{k}\right|\right]$, the following equation holds approximately:

$$
\boldsymbol{P}_{x_{k+1} y_{k+1}}-\boldsymbol{K}_{k+1} \boldsymbol{V}_{0, k+1}=0 .
$$


Therefore, we can choose the appropriate time-varying gain matrix $\boldsymbol{K}_{k+1}$ to make (23) hold. From the UKF formula we can obtain the following equation:

$$
\boldsymbol{P}_{x_{k+1} y_{k+1}}\left(\boldsymbol{I}-\boldsymbol{P}_{y_{k+1} y_{k+1}}^{-1} \boldsymbol{V}_{0, k+1}\right)=0
$$

thus

$$
\boldsymbol{P}_{y_{k+1} y_{k+1}}=\boldsymbol{V}_{0, k+1} \text {. }
$$

A sub-optimal factor $\lambda_{k+1}$ is utilized to make the following equation hold:

$$
\begin{gathered}
\lambda_{k+1} \sum_{i=0}^{2 n} W_{i}\left[\boldsymbol{\gamma}_{i, k+1 \mid k}-\hat{\boldsymbol{y}}_{k+1 \mid k}\right]\left[\boldsymbol{\gamma}_{i, k+1 \mid k}-\hat{\boldsymbol{y}}_{k+1 \mid k}\right]^{\mathrm{T}}= \\
{\left[\boldsymbol{V}_{0, k+1}-\boldsymbol{R}_{k+1}\right] .}
\end{gathered}
$$

And the parameter $\lambda_{k+1}$ is calculated from (27)-(33) [26]:

$$
\begin{gathered}
\boldsymbol{\gamma}_{k+1}=\boldsymbol{Y}_{k+1}-\hat{\boldsymbol{Y}}_{k+1 \mid k} \\
\boldsymbol{V}_{0, k+1}=\left\{\begin{array}{l}
\gamma_{1} \gamma_{1}^{\mathrm{T}}, \quad k=0 \\
\frac{\rho \boldsymbol{V}_{0, k}+\gamma_{k+1} \boldsymbol{\gamma}_{k+1}^{\mathrm{T}}}{1+\rho}, \quad k \geqslant 1
\end{array}\right. \\
\boldsymbol{M}_{k+1}=\sum_{i=0}^{2 n} W_{i}\left[\boldsymbol{\gamma}_{i, k+1 \mid k}-\hat{\boldsymbol{y}}_{k+1 \mid k}\right] . \\
{\left[\boldsymbol{\gamma}_{i, k+1 \mid k}-\hat{\boldsymbol{y}}_{k+1 \mid k}\right]^{\mathrm{T}}} \\
\boldsymbol{N}_{k+1}=\operatorname{tr}\left[\boldsymbol{V}_{0, k+1}-\boldsymbol{R}_{k+1}\right] \\
c_{k+1}=\frac{\operatorname{tr}\left(\boldsymbol{N}_{k+1}\right)}{\operatorname{tr}\left(\boldsymbol{M}_{k+1}\right)}
\end{gathered}
$$

where $c_{k+1}$ is a factor to be determined as follows:

$$
\begin{aligned}
\lambda_{k+1} & =\left\{\begin{array}{l}
\alpha c_{k+1}, \quad \vartheta \leqslant c_{k+1} \\
c_{k+1}, \quad 1 \leqslant c_{k+1} \\
1, \quad c_{k+1} \leqslant 1
\end{array}\right. \\
\lambda_{i, k+1} & =\alpha_{i} c_{k+1}, \quad i=1,2, \ldots, n
\end{aligned}
$$

where $\alpha, \vartheta$ are pre-defined constants.

The strong tracking filter can be utilized in the adaptive UKF. The sub-optimal fading factor $\lambda_{k+1}$ which is used to fade the past data and adjust the predicted state estimation covariance matrix, is calculated from (27)-(33). The standard UKF becomes a strong tracking filter when (19) is changed as follows:

$$
\boldsymbol{P}_{k+1}=\lambda_{k+1} \boldsymbol{P}_{k+1 \mid k}-\boldsymbol{K}_{k+1} \boldsymbol{P}_{y_{k+1} y_{k+1}} \boldsymbol{K}_{k+1}^{\mathrm{T}} .
$$

\subsection{Estimation of measurement sequence noise}

When the statistics of measurement noise are not accurate, the filtering performance will seriously deteriorate [27], even for some high precision non-linear filters, such as the UKF. When the parameter setting is not appropriate, its filtering accuracy is even lower than the normal EKF. Sage and Husa proposed the Sage-Husa adaptive noise filtering algorithm [28] to estimate the noise, but the filtering algorithm cannot guarantee stability and convergence [29]. At the same time, many model-based noise estimation methods were proposed, however, their tracking abilities are not enough for the estimation of mutation parameters, states and noise [30,31]. Wavelet transform estimation can estimate the change noise accurately and robustly without the information of the system noise models.

Wavelet transform, utilized widely in data processing, signal de-noising, and image processing, has a good estimation of the noise and processing power [32,33]. A wavelet transform can be used to estimate the variance of the noise in a fast time-varying signal [34]. If the wavelet is carefully selected, the estimation of the variance may not be influenced by the fluctuation of the fast time-varying signal [35].

Therefore, a wavelet transform is utilized to estimate the statistical information of the measurement noise in this subsection. We only need to estimate the noise variance, regardless of the mean [36].

Measurement data with length $L$ are employed for the wavelet transform.

The $m$-dimensional measurement sequence $y_{i}(t)$ which satisfies (2) is

$$
y_{i}(t)=h(t)+d_{i}(t), \quad i=1,2, \ldots, m .
$$

To estimate the variance $\sigma^{2}$ of noise $d(t)$ from the data $y(t)=h(t)+d(t)$, we need to suppress the influence of $h(t)$. When $h$ is piecewise smooth, a robust estimator is calculated from the median of the finest scale wavelet coefficients [37].

It is supposed that $\psi(t) \in L^{2}(\mathbf{R})$ is a wavelet base function and its Fourier transform is $\hat{\psi}(\omega)$, which satisfies the perfect reconstruction condition as follows:

$$
\begin{gathered}
C_{\psi}=\int_{-\infty}^{\infty} \frac{|\hat{\psi}(\omega)|^{2}}{|\omega|} \mathrm{d} \omega<\infty \\
\psi_{a, b}(t)=\frac{1}{\sqrt{|a|}} \psi\left(\frac{t-b}{a}\right), \quad a, b \in \mathbf{R} ; a \neq 0
\end{gathered}
$$

which is the dilation and translation of $\psi(t)$, where $a$ is the scaling factor and $b$ is the transforming factor.

The wavelet transform for the function $y_{i}(t)$ at time $b$ and scale $a$ is

$$
\begin{gathered}
\boldsymbol{W}_{y_{i}}(a, b)=\left\langle y_{i}, \psi_{a, b}\right\rangle=|a|^{-\frac{1}{2}} \int_{-\infty}^{\infty} y_{i}(t) \overline{\psi\left(\frac{t-b}{a}\right)} \mathrm{d} t \\
i=1,2, \ldots, m
\end{gathered}
$$


Because the wavelet transform has linear characteristics, the wavelet transform can be written as

$$
\boldsymbol{W}_{y_{i}}(a, b)=\boldsymbol{W}_{h_{i}(t)}(a, b)+\boldsymbol{W}_{d_{i}(t)}(a, b) .
$$

If $h(t)$ can be described in the following form:

$$
h(t)=\sum_{j=0}^{M} a_{i} t^{i}
$$

and $\psi(t)$ has $p(p>M)$ vanishing moments:

$$
\int_{-\infty}^{+\infty} t^{k} \psi(t) \mathrm{d} t=0, \quad 0 \leqslant k<p
$$

the wavelet transform of $y_{i}(t)$ suppresses signal $h(t)$ and retains the noisy component $d_{i}(t)$ only. Similar to [34], (39) becomes

$$
\boldsymbol{W}_{y_{i}}(a, b)=\boldsymbol{W}_{d_{i}(t)}(a, b) .
$$

A robust estimator of $\sigma^{2}$ is calculated from the median of $\left\{\boldsymbol{W}_{y_{i}}(a, b)\right\}_{0 \leqslant b<N / 2}$ which is the $N / 2$ wavelet coefficient of $y_{i}(k)(k=1, \ldots, N)$, the discrete representation of $y_{i}(t)$.

Typically, as the number of measurements is always limited, we can only obtain a valuation of standard deviation $\sigma$, denoted by $M$ and termed as "mean square error", and its value can be calculated as follows:

$$
\hat{\sigma}=M
$$

The standard deviation $\sigma$ of variables satisfies Gaussian distribution and can be estimated by the probable error $\rho_{p}$. Due to the fact that the number of measurements is limited, the valuation of $\rho_{p}$ will be denoted by $\omega$ which is called the probable error. The probable error and the mean square error satisfy the following relationship:

$$
\omega \approx 0.6745 M
$$

so

$$
\hat{\sigma} \approx \frac{\omega}{0.6745}=\frac{\operatorname{median}\left|\boldsymbol{W}_{y_{i}}(a, b)\right|}{0.6745}
$$

where median $\left|\boldsymbol{W}_{y_{i}}(a, b)\right|$ is the middle value of the data sequence $\left|\boldsymbol{W}_{y_{i}}(a, b)\right|[35,37-39]$.

The length of the most recent data used for variance estimation must be limited because the statistical properties of the measurement noise are not fixed. This length is termed as the estimation length and denoted by $L$. The length $L$ depends on the sampling frequency and the response time [35]:

$$
L=\tau f_{s}
$$

where $\tau$ is response time, and $f_{s}$ is the sampling frequency.
The value of length $L$ should be as large as possible, depending on the computational burden and the noise level. Therefore, $L$ is a trade-off between the precision and the calculation speed, as well as a compromise between sensitivity and robustness of the choice, satisfying the following expression:

$$
\left\{\begin{array}{l}
\min _{L} \delta_{a}=\sum_{k=1}^{n}\left(\left|\hat{a}_{i}(k)-a_{i}(k)\right|\right) \\
\max L, \quad L \in[1, n] \\
\min T
\end{array}\right.
$$

where $n$ is the number of sampling points, $T$ is the time of calculation, $\hat{a}_{i}(k)$ is the estimation value of parameters, and $a_{i}(k)$ is the true value of parameters.

\subsection{Stability improvement}

The general UKF may not be stable, especially in a highdimensional system. The inverse operation makes some large elements of the covariance matrix become very small. After a few operations, the elements which are close to zero become zero due to truncation errors. The accumulation and spread of truncation errors usually destroy the positive definiteness essential to a covariance matrix and lead to the instability of numerical calculations in the general UKF.

Because the nonlinear high-speed objects are always described by a high-dimensional mathematical model. We introduce square-root forms of the strong tracking UKF (STUKF) to improve the stability of STUKF. The squareroot STUKF has better numerical properties and guarantees positive semi-definiteness of the underlying state covariance. In addition, the square-root STUKF and some efficient EKF have the same complexity in the parameter estimation. Furthermore, we also use the augmented form to improve the accuracy of STUKF.

The augmented square-root STUKF is as follows [40]:

(i) Compute the initialization, sigma points and weights

$$
\begin{gathered}
\hat{\boldsymbol{x}}_{0}=\mathrm{E}\left[\boldsymbol{x}_{0}\right] \\
\boldsymbol{S}_{x_{0}}=\operatorname{chol}\left\{\mathrm{E}\left[\left(\boldsymbol{x}_{0}-\hat{\boldsymbol{x}}_{0}\right)\left(\boldsymbol{x}_{0}-\hat{\boldsymbol{x}}_{0}\right)^{\mathrm{T}}\right]\right\} \\
\boldsymbol{S}_{Q}=\sqrt{\boldsymbol{Q}} \\
\boldsymbol{S}_{R}=\sqrt{\boldsymbol{R}} \\
\boldsymbol{S}_{x_{0}}^{a}=\operatorname{chol}\left\{\mathrm{E}\left[\left(\boldsymbol{x}_{0}^{a}-\hat{\boldsymbol{x}}_{0}^{a}\right)\left(\boldsymbol{x}_{0}^{a}-\hat{\boldsymbol{x}}_{0}^{a}\right)^{\mathrm{T}}\right]\right\}= \\
{\left[\begin{array}{ccc}
\boldsymbol{S}_{x_{0}} & 0 & 0 \\
0 & \boldsymbol{S}_{Q} & 0 \\
0 & 0 & \boldsymbol{S}_{R}
\end{array}\right]} \\
\chi_{k}\left[\hat{\boldsymbol{x}}_{k}, \hat{\boldsymbol{x}}_{k}+\sqrt{\varpi} \boldsymbol{S}_{x_{k}}^{a}, \hat{\boldsymbol{x}}_{k}-\sqrt{\varpi} \boldsymbol{S}_{x_{k}}^{a}\right] \\
W_{0}^{(m)}=\eta / \varpi
\end{gathered}
$$




$$
\begin{gathered}
W_{0}^{(c)}=\eta / \varpi+\left(1-\alpha^{2}+\beta\right) \\
W_{i}^{(m)}=W_{i}^{(c)}=\varpi / 2, \quad i=1, \ldots, 2 n \\
\eta=\alpha^{2}(n+\kappa)-n
\end{gathered}
$$

(ii) Time update

$$
\begin{aligned}
& \chi_{k+1 \mid k}=\boldsymbol{f}\left(\chi_{k}\right) \\
& \gamma_{k+1 \mid k}=\boldsymbol{h}\left(\chi_{k+1 \mid k}\right) \\
& \hat{\boldsymbol{x}}_{k+1 \mid k}=\sum_{i=0}^{2 n} W_{i}^{(m)} \chi_{i, k+1 \mid k} \\
& \boldsymbol{S}_{x_{k+1 \mid k}}=\operatorname{qr}\left\{\left[\sqrt{W_{1}^{(c)}}\left(\boldsymbol{\chi}_{1: 2 n, k+1 \mid k}-\hat{\boldsymbol{x}}_{k+1 \mid k}\right)\right]\right\} \\
& \boldsymbol{S}_{x_{k+1 \mid k}}=\text { cholupdate }\left\{\boldsymbol{S}_{x_{k+1 \mid k}}, \boldsymbol{\chi}_{0, k+1 \mid k}-\hat{\boldsymbol{x}}_{k+1 \mid k}, W_{0}^{(c)}\right\} \\
& \hat{\boldsymbol{y}}_{k+1 \mid k}=\sum_{i=0}^{2 n} W_{i}^{(m)} \boldsymbol{\gamma}_{i, k+1 \mid k} \\
& \boldsymbol{S}_{y_{k}}=\operatorname{qr}\left\{\left[\sqrt{W_{1}^{(c)}}\left(\gamma_{1: 2 n, k+1 \mid k}-\hat{\boldsymbol{y}}_{k+1 \mid k}\right)\right]\right\} \\
& \boldsymbol{S}_{y_{k}}=\text { cholupdate }\left\{\boldsymbol{S}_{y_{k}}, \gamma_{0, k+1 \mid k}-\hat{\boldsymbol{y}}_{k+1 \mid k}, W_{0}^{(c)}\right\} \\
& \boldsymbol{P}_{x_{k+1} y_{k+1}}=\sum_{i=0}^{2 n} W_{i}^{(c)}\left[\chi_{i, k+1 \mid k}-\hat{\boldsymbol{x}}_{k+1 \mid k}\right] \text {. } \\
& {\left[\gamma_{i, k+1 \mid k}-\hat{\boldsymbol{y}}_{k+1 \mid k}\right]^{\mathrm{T}}} \\
& \boldsymbol{K}_{k+1}=\left(\boldsymbol{P}_{x_{k+1} y_{k+1}} / \boldsymbol{S}_{y_{k}}^{\mathrm{T}}\right) / \boldsymbol{S}_{y_{k}} \\
& \hat{\boldsymbol{x}}_{k+1}=\hat{\boldsymbol{x}}_{k+1 \mid k}+\boldsymbol{K}_{k+1}\left(\boldsymbol{y}_{k+1}-\hat{\boldsymbol{y}}_{k+1 \mid k}\right) \\
& \boldsymbol{U}=\boldsymbol{K}_{k+1} \boldsymbol{S}_{y_{k}} \\
& \boldsymbol{S}_{x_{k}}=\operatorname{cholupdate}\left\{\sqrt{\lambda_{k+1}} \boldsymbol{S}_{x_{k+1 \mid k}}, \boldsymbol{U},-1\right\}
\end{aligned}
$$

where $\operatorname{qr}\{[\cdot]\}$ is the QR decomposition of $[\cdot]$, cholupdate $\{\boldsymbol{S}, \boldsymbol{U}, \pm \nu\}$ is the Cholesky factor updating of covariance matrix which means $\lambda_{k+1} \boldsymbol{P} \pm \sqrt{\nu} \boldsymbol{U} \boldsymbol{U}^{\mathrm{T}}, \boldsymbol{S}$ is the Cholesky factor of $\boldsymbol{P}$, and the factor $\lambda_{k+1}$ is derived by (27)-(33).

\subsection{Implementation of the algorithm}

The algorithm can be implemented by the following procedure:

(i) Calculate $\hat{\sigma}$ using (45). According to the computation time and computational complexity of the preliminary estimate, select an appropriate length $L$, an appropriate mother wavelet function, and estimate $\boldsymbol{R}_{k+1}$ by the following formula:

$$
\boldsymbol{R}_{k+1}=\left[\begin{array}{cccc}
\hat{\sigma}_{1}^{2} & 0 & \cdots & 0 \\
0 & \hat{\sigma}_{2}^{2} & \cdots & 0 \\
\vdots & \vdots & \ddots & \vdots \\
0 & 0 & 0 & \hat{\sigma}_{m}^{2}
\end{array}\right]
$$

(ii) For estimating the nonlinear parameter $\theta_{k}$, get an augmented state including $\boldsymbol{x}_{k}$ and $\theta_{k}$ in (3) and an augmented noise in (4).

(iii) Use the adaptive UKF to estimate the parameter $\theta_{k}$ or the state, from (48) to (69). Considering the stability and accuracy of calculations, we suggest that the value of $\alpha$ is from 0.7 to 1 .

Remark 1 Further improvement in calculation speed-

\section{RUKF}

In some cases, if necessary, we can improve the calculation speed further. We can take the following form to reduce sigma points $[23,41]$ named reduced sigma points UKF (RUKF). The weights of sigma points in RUKF are as follows:

$$
\begin{gathered}
0 \leqslant W_{0}<1 \\
W_{i}=\left\{\begin{array}{l}
\frac{1-W_{0}}{2^{n}}, \quad i=1 \\
W_{1}, \quad i=2 \\
2^{i-2} W_{1}, \quad i=3, \ldots, n+1
\end{array}\right.
\end{gathered}
$$

where $W_{0}$ is a free parameter whose value affects the fourth and higher moments of the sigma point set. We suggest that the value of $W_{0}$ is close to 1 .

Initialize the vector sequence as:

$$
\chi_{0}^{1}=[0], \chi_{1}^{1}=\left[-\frac{1}{\sqrt{2 W_{1}}}\right], \chi_{2}^{1}=\left[\frac{1}{\sqrt{2 W_{1}}}\right] .
$$

Expand the vector sequence for $j=2, \ldots, n$ according to

$$
\chi_{i}^{j}=\left\{\begin{array}{l}
{\left[\begin{array}{c}
\chi_{0}^{j-1} \\
0
\end{array}\right], i=0} \\
{\left[\begin{array}{c}
\chi_{i}^{j-1} \\
\left.-\frac{1}{\sqrt{2 W_{j}}}\right], i=1, \ldots, j . \\
{\left[\frac{1}{0_{j-1}}\right], i=j+1}
\end{array}\right]}
\end{array}\right.
$$

The next calculation is the same as the general UKF.

Remark 2 Non-additive noise case

For non-additive noise or general noise, the system equations are as follows:

$$
\begin{gathered}
\boldsymbol{x}_{k+1}=\boldsymbol{f}\left(\boldsymbol{x}_{k}, \boldsymbol{v}_{k}\right) \\
\boldsymbol{y}_{k+1}=\boldsymbol{h}\left(\boldsymbol{x}_{k+1}, \boldsymbol{w}_{k+1}\right)
\end{gathered}
$$

where $\boldsymbol{v}$ and $\boldsymbol{w}$ are the process noise and the measurement noise, respectively, and their covariance matrices are $\boldsymbol{Q}_{k}$ and $\boldsymbol{R}_{k+1}$, respectively.

We can augment the measurement and process noise into states using (51), then we can use the adaptive UKF to estimate states and parameters. 
Remark 3 High-dimensional numerical stability

In some extreme cases such as those with high dimension and too large noise, the covariance matrix may be non-positive definite. The Cholesky decomposition of the covariance matrix may not proceed. Some methods are available to solve this problem. The predicted covariance matrix is calculated with enlarged $Q_{k}+\Delta Q_{k}$ [42], where $\Delta \boldsymbol{Q}_{k}$ is an extra positive definite matrix introduced in the calculated covariance matrix as a slight modification of the UKF so that the stability will be improved.

\section{Numerical example}

We take the same example from [26] in order to compare the proposed filter with the traditional strong tracking EKF and the general UKF:

$$
\left\{\begin{array}{rl}
x_{1}(k+1)= & m_{1} a(k) x_{1}^{2}(k)+x_{1}(k)+ \\
& m_{1} x_{2}(k) u(k)+\nu_{1}(k) \\
x_{2}(k+1)= & x_{2}(k)+\nu_{2}(k) \\
y_{1}(k+1)= & a^{3}(k+1) x_{1}(k+1)+e_{1}(k+1) \\
y_{2}(k+1)= & m_{2} x_{1}^{2}(k+1)+e_{2}(k+1)
\end{array} .\right.
$$

The true value of $a(k)$ to be estimated is a square wave with a $200 \mathrm{~s}$ period and a $1000 \mathrm{~s}$ duration. The sampling time of the square wave is $1 \mathrm{~s}$. The noise is Gaussian white noise. Take $x_{1}(0)=0, x_{2}(0)=0.2, a(0)=0, \boldsymbol{P}(0 \mid 0)=$ $0.0001 \boldsymbol{I}, m_{1}=0.1, m_{2}=4.5$. The parameters of the general strong tracking EKF (STEKF) are as follows: $\vartheta=5$, $\rho=0.95$. For UKF and adaptive UKF, $\alpha=0.7, \beta=$ $2, \kappa=0, \boldsymbol{R}_{0}=\operatorname{diag}\left\{0.002^{2}, 0.002^{2}\right\}$, the matching process noise $\boldsymbol{Q}_{m}^{a}=\operatorname{diag}\left\{0.002^{2}, 0.002^{2}, 0.002^{2}\right\}$.

The true values of input variable $u$, states $\boldsymbol{x}$ and output $\boldsymbol{y}$ are shown in Fig. 1.

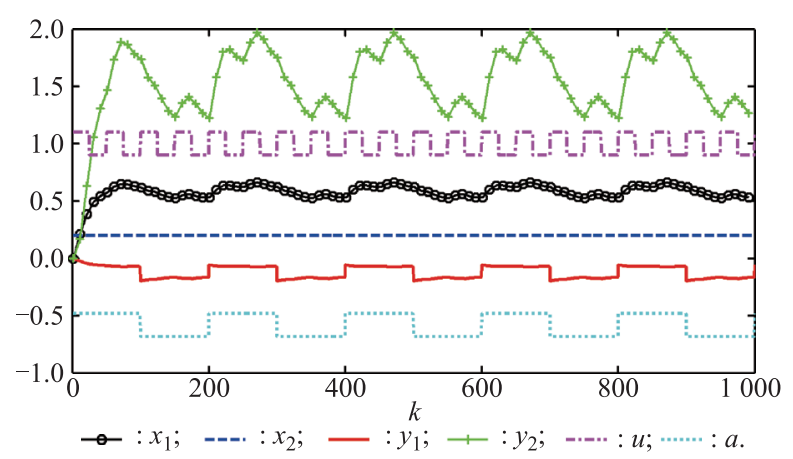

Fig. 1 True values of states and parameters

In order to compare the AUKF with the traditional strong tracking EKF and the general UKF, we set a noise matching UKF which has the same variances of noises as simulation settings. In the first numerical simulation, we increase the values of the measurement noise variance by 100 times in 200-399 s and 600-799 s, and the STEKF, the general UKF, the noise matching UKF (mUKF) and the AUKF are utilized to estimate the parameter $a(k)$, respectively. The results are shown in Fig. 2.

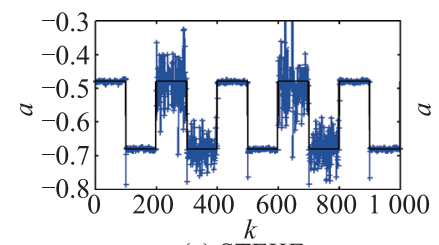

(a) STEKF

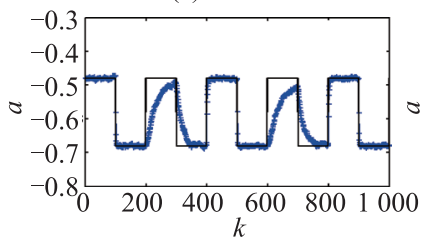

(c) $\mathrm{mUKF}$

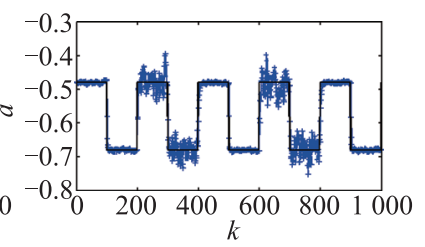

(b) UKF

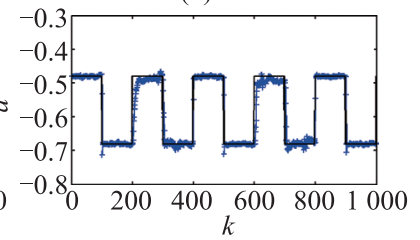

(d) AUKF
Fig. 2 Parameter estimation results

Fig. 2 illustrates that even if the variances of mUKF are consistent with settings of simulations, the estimated results of mUKF are still not satisfied due to limitation of tracking ability for mutation parameters, and AUKF has high-precision, strong tracking ability and adapts to changes in measurement noise capabilities.

We take the accumulation of the absolute state estimation error and the absolute parameter estimation error value as the performance index:

$$
\begin{gathered}
\delta_{X}=\sum_{i=1}^{N} \sum_{k=1}^{m}\left(\left|\hat{x}_{i}(k)-x_{i}(k)\right|\right) \\
\delta_{a}=\sum_{k=1}^{N}\left(\left|\hat{a}_{i}(k)-a_{i}(k)\right|\right)
\end{gathered}
$$

where $N$ is the iteration and $m$ is the number of states.

The means of 100 times Monte Carlo simulations of $\delta_{X}$ and $\delta_{a}$ is shown in Table 1 .

Table 1 Comparison of estimated results

\begin{tabular}{ccccc}
\hline & STEKF & UKF & mUKF & AUKF \\
\hline$\delta_{X}$ & 81.922 & 68.103 & 79.944 & 66.330 \\
$\delta_{a}$ & 20.487 & 13.675 & 25.501 & 11.034 \\
\hline
\end{tabular}

Table 1 illustrates that mUKF lacks the tracking ability, and the STEKF and UKF cannot adapt to changes in the measurement noise, so AUKF is better than the other methods in estimating states and parameters.

In the second simulation, the process noise $\boldsymbol{Q}_{k}^{a}$ is set up inaccurately. The other conditions are the same as the first simulation. After 100 times Monte Carlo simulations, the estimation results of parameters and states using STEKF, UKF, mUKF and AUKF are shown in Fig. 3 and 
Fig. 4. Fig. 3 gives estimation results when process noise $\boldsymbol{Q}_{k}^{a}=0.01 \boldsymbol{Q}_{m}^{a}$. Fig. 4 gives estimation results when process noise $\boldsymbol{Q}_{k}^{a}$ is changed from $0.01 \boldsymbol{Q}_{m}^{a}$ to $100 \boldsymbol{Q}_{m}^{a}$.

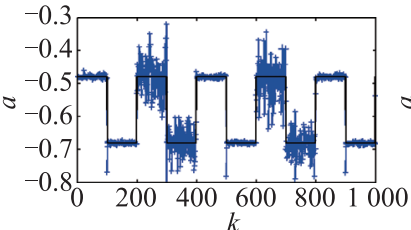

(a) STEKF

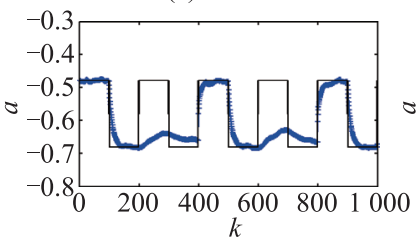

(c) $\mathrm{mUKF}$
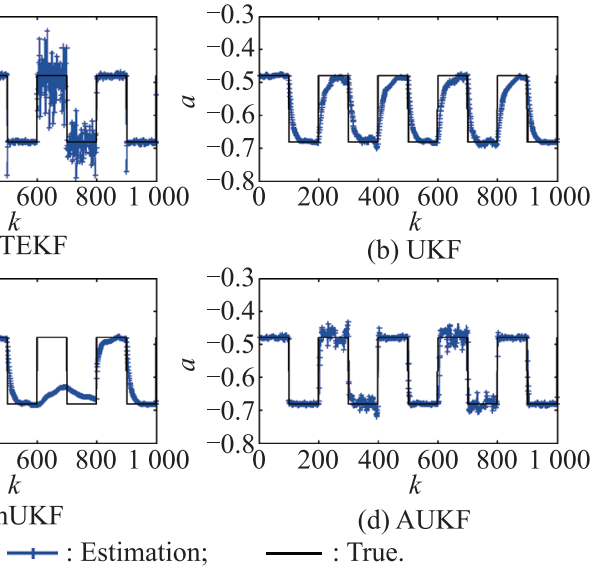

(b) UKF

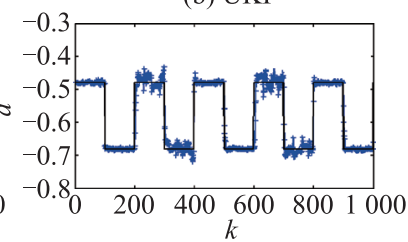

(d) AUKF

: True.

Fig. 3 Parameter estimation results when $Q_{k}^{a}$ is inaccurate
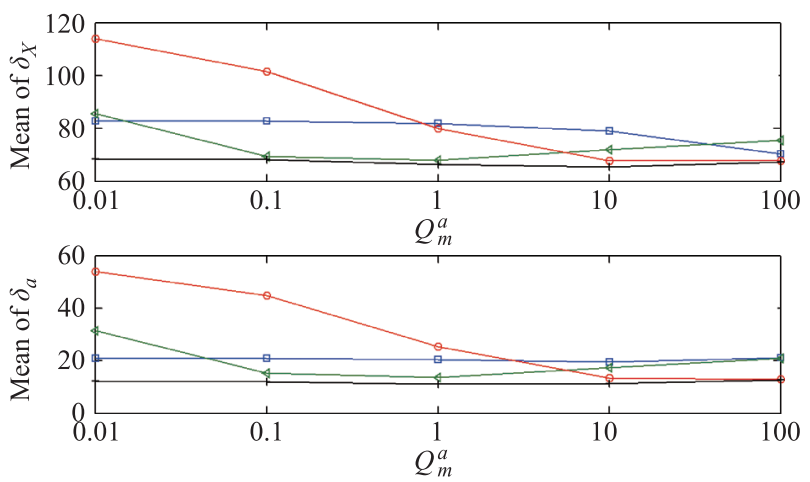

$\neg:$ STEKF; $\triangleleft:$ UKF; $\bullet:$ mUKF; — $:$ AUKF.

Fig. 4 Estimation results when $\boldsymbol{Q}_{k}^{a}$ is changed from $0.01 \boldsymbol{Q}_{m}^{a}$ to $100 \boldsymbol{Q}_{m}^{a}$

Fig. 3 and Fig. 4 illustrate the robustness of AUKF against the inaccurate process noise and the changed measurement noise, indicating that AUKF preforms better than general UKF, mUKF and STEKF.

\section{Application in estimation of artillery shell}

In this section, we use the AUKF method to estimate the ballistic parameters and states, comparing it with EKF, STEKF, the general UKF and the reduced sigma points UKF (RUKF). Ballistic equation is as follows [43,44]:

$$
\left\{\begin{array}{l}
\frac{\mathrm{d} V_{x}}{\mathrm{~d} t}=-\rho_{m} C_{1} V V_{x} \\
\frac{\mathrm{d} V_{y}}{\mathrm{~d} t}=-\rho_{m} C_{1} V V_{y}-g_{0} \\
\frac{\mathrm{d} V_{z}}{\mathrm{~d} t}=-\rho_{m} C_{1} V V_{z}, \quad \frac{\mathrm{d} x}{\mathrm{~d} t}=u_{x} \\
\frac{\mathrm{d} y}{\mathrm{~d} t}=u_{y}, \quad \frac{\mathrm{d} z}{\mathrm{~d} t}=u_{z}
\end{array}\right.
$$

The initial values of differential equation are

$$
\begin{gathered}
\left\{\begin{array}{l}
u_{x}=u_{0} \cos \theta_{0} \\
u_{y}=u_{0} \sin \theta_{0} \\
u_{z}=0 \\
x=L_{0} \cos \theta_{0} \\
y=y_{0}+L_{0} \sin \theta_{0} \\
z=0
\end{array}\right. \\
V=\sqrt{V_{x}^{2}+V_{y}^{2}+V_{z}^{2}}= \\
\sqrt{\left(u_{x}-W_{x}\right)^{2}+\left(u_{y}-W_{y}\right)^{2}+\left(u_{z}-W_{z}\right)^{2}}
\end{gathered}
$$

where $C_{1}=\frac{S}{2 m} F_{D} C_{D_{0}}, V_{x}, V_{y}, V_{z}$ are the values of speed in $x, y$ and $z$ directions, and $x, y, z$ are position coordinates; $C_{1}$ is the comprehensive ballistic trajectory coefficient; $C_{D_{0}}$ is the zero drag coefficient; $F_{D}$ is the marching drag coefficient; $m$ is the weight of shell; $S$ is the reference area of shell; and $\rho_{m}$ is the air density which is a nonlinear function of height $h$ :

$$
\begin{aligned}
& \rho_{m}=\left\{\begin{array}{l}
0.2063, \quad h<0 \\
0.2063(1-0.000021905 h)^{4.4} \\
0 \leqslant h \leqslant 9300 \\
\frac{0.3525713325 \tau_{o n} \mathrm{e}^{-2.1206426\left[\Xi_{2}\right]}}{\Xi_{1}}, \\
9300<h \leqslant 12000 \\
\frac{0.2336904675 \tau_{o n} \mathrm{e}^{-\frac{h-12000}{6483.305}}}{\Xi_{1}} \\
\Xi_{1}=230+0.006328(h-9300)+ \\
0.000001172(h-9300)^{2}
\end{array}\right. \\
& \Xi_{2}=\arctan \left(\frac{2.344(h-9300)-6328}{32221.057}\right)+0.1939252
\end{aligned}
$$

where $\tau_{o n}$ is the virtual temperature of the ground, and the value of $\tau_{\text {on }}$ is usually $288.9 \mathrm{~K}$. The state vectors are selected as

$$
\begin{gathered}
\boldsymbol{X}=\left[x_{1}, x_{2}, x_{3}, x_{4}, x_{5}, x_{6}, x_{7}\right]^{\mathrm{T}}= \\
{\left[V_{x}, V_{y}, V_{z}, x, y, z, C_{1}\right]^{\mathrm{T}} .}
\end{gathered}
$$

Rewrite (81), we can get

$$
\dot{\boldsymbol{X}}=\boldsymbol{f}(\boldsymbol{X})=\left[\begin{array}{c}
-\rho_{m} x_{1} x_{7} \sqrt{x_{1}^{2}+x_{2}^{2}+x_{3}^{2}} \\
-\rho_{m} x_{2} x_{7} \sqrt{x_{1}^{2}+x_{2}^{2}+x_{3}^{2}}-g_{0} \\
-\rho_{m} x_{3} x_{7} \sqrt{x_{1}^{2}+x_{2}^{2}+x_{3}^{2}} \\
x_{1} \\
x_{2} \\
x_{3} \\
0
\end{array}\right]+\boldsymbol{\Upsilon}
$$


Nonlinear equation (81) is only an inaccurate description of the ballistic trajectory, and its approximation error could be modeled by zero-mean Gaussian white noise $\boldsymbol{r}$. Select the speed as a measurement, and the measurement equation is:

$$
\boldsymbol{y}=\boldsymbol{h}(\boldsymbol{x})+\boldsymbol{w}=\left[\begin{array}{l}
x_{1} \\
x_{2} \\
x_{3}
\end{array}\right]+\boldsymbol{w}
$$

where $\boldsymbol{w}$ is the zero mean Gaussian white measurement noise, the covariance of $\boldsymbol{w}$ is $\sigma^{2}$.

Artillery shell is a typical nonlinear high-speed object which has the nonlinear high-speed objects features mentioned in Section 2 and also has the following characteristics:

First, the movement disciplinary of the artillery shell is always described by a group of trajectory equations which are a class of continuous differential equations. The differential equations do not have exact analytical solutions but numerically approximate solutions.

Second, the trajectory parameters of the equation are nonlinear time-varying parameters, and most of them are related to the speed. The piecewise expressions are utilized to fit and approximate the ballistic parameters. Take (81) as an example, the trajectory parameter $C_{1}$ is a speed-related parameter, and the parameter is a function of other parameters. Therefore, the expression for the parameters of trajectory equations is very complex, and it is difficult to derive the Jacobian matrix.

In addition, there is a strong coupling between state variables and parameters. In (81), the air density $\rho_{m}$ is a nonlinear parameter depending on the temperature and altitude, and the temperature is related to the height which is related to the speed. The expression of density $\rho_{m}$ is given by (84), and its schematic diagram is shown in Fig. 5.

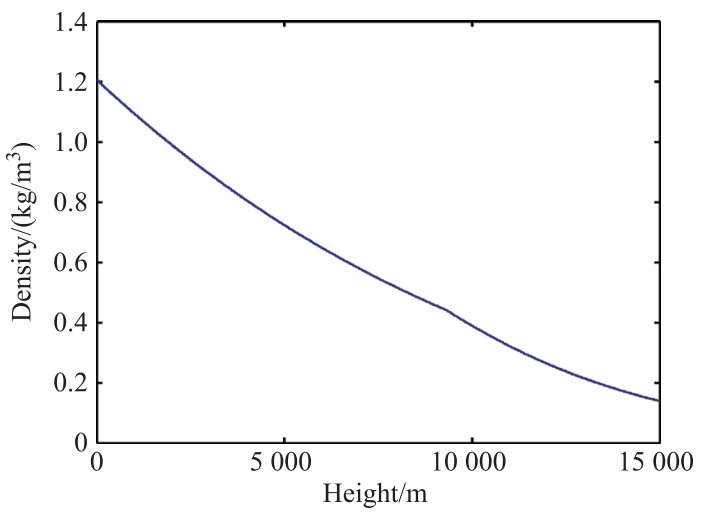

Fig. 5 Relationship between density and height in trajectory equation

We compare EKF, STEKF, the general UKF, RUKF with AUKF filters for the state estimation and parame- ter identification of the artillery shell. In the calculation, we take velocity values as measurements, which are the 0-25 s part of the whole artillery shell projectile, and measurement noise variance changes as shown in Table 2. $T$ denotes the calculation time.

Table 2 Measurement noise variance change of ballistic trajectory

\begin{tabular}{cccccc}
\hline$T / \mathrm{s}$ & $0-5$ & $5-10$ & $10-15$ & $15-20$ & $20-25$ \\
\hline$\sigma^{2}$ & 0.00001 & 0.002 & 0.01 & 0.002 & 0.00001 \\
\hline
\end{tabular}

Take $\vartheta=5, \rho=0.95, \alpha=0.9999, \beta=2, \kappa=-4$, $W_{0}=0.9999, \boldsymbol{P}(0 \mid 0)=\operatorname{diag}\left\{1,1,1,1,1,1,10^{-9}\right\}$, $\boldsymbol{Q}=\operatorname{diag}\left\{0.01,0.01,0.01,0.01,0.01,0.01,10^{-10}\right\}$, $\boldsymbol{X}(0)=[694.27,139.25,0,0,0,0,0.00076]^{\mathrm{T}}$. The means of 100 times simulations is shown in Table $3 . \delta_{X}$ is the accumulative absolute estimation error of states. $\delta_{C_{1}}$ is the accumulative absolute estimation error of parameter $C_{1}$.

Table 3 Comparison of estimated results

\begin{tabular}{cccccc}
\hline & EKF & STEKF & UKF & RUKF & AUKF \\
\hline$\delta_{X}$ & 11220.00 & 11184.00 & 5956.20 & 6958.90 & 5911.80 \\
MSE & 167.16 & 166.85 & 42.37 & 54.20 & 41.97 \\
$\delta_{C_{1}}\left(10^{-4}\right)$ & 4.16 & 3.93 & 4.38 & 9.31 & 3.02 \\
$T / \mathrm{s}$ & 0.0249 & 0.0298 & 0.2063 & 0.0577 & 0.1234 \\
\hline
\end{tabular}

The estimation results of $C_{1}$ are shown in Fig. 6.

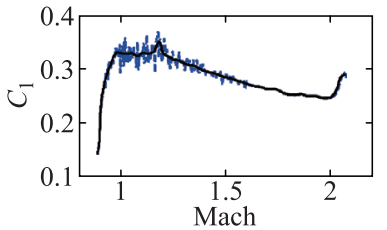

(a) STEKF

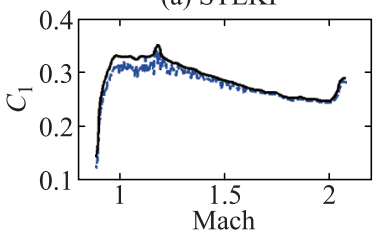

(c) RUKF

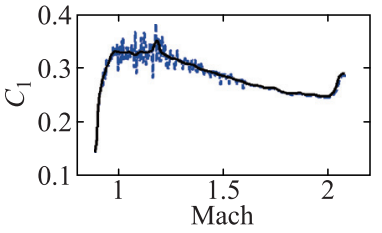

(b) UKF

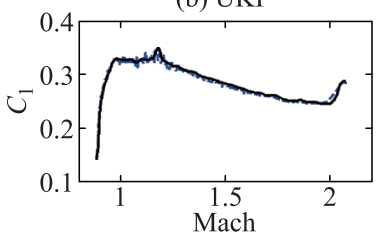

(d) AUKF
Fig. 6 Comparison of parameter estimation results

From Fig. 6, EKF, general UKF and RUKF cannot adapt to changes in the measurement noise and have big errors. AUKF results are still good, though the measurement noise has changed greatly.

The accumulative errors $\delta_{a}$ in 100 times simulations are shown in Fig. 7. The simulations were implemented on a PC with $3 \mathrm{GHz}$ Pentium IV CPU and 1 GB internal memories.

From Fig. 7, the parameter estimation accuracy of AUKF is always better than those of the other four methods.

The simulation results from Fig. 6, Fig. 7 and Table 3 show that the AUKF is better than EKF, STEKF, general UKF and RUKF in terms of parameter and state estimation accuracy and computational stability. The computing time 


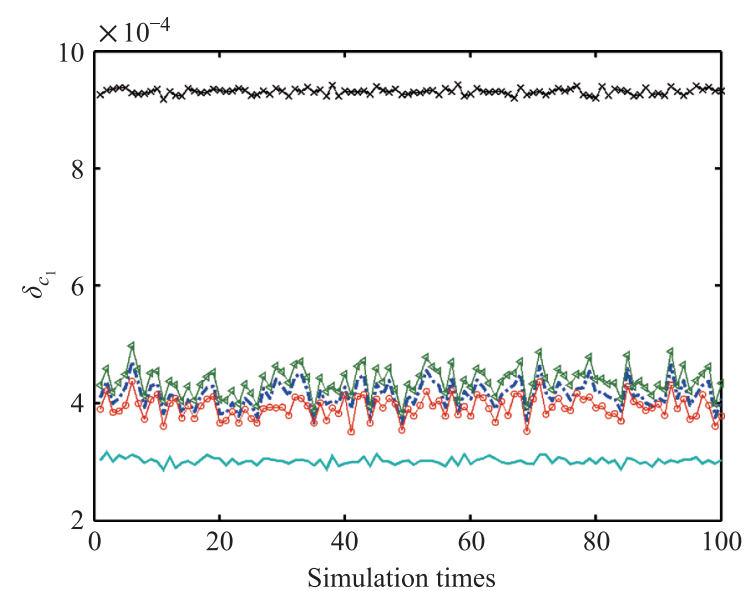

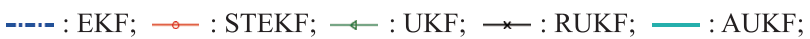

Fig. 7 Accumulated error of 100 simulations for parameter estimation

of AUKF is less than that of the general UKF and meets the time requirements of trajectory estimation. In practice, we use a dedicated DSP based parallel computing platform. The signal noise estimation, parameter and state estimation are processed in parallel, so the computing speed of the system are greatly increased.

\section{Conclusions}

A method for the estimation of parameters and states of high-speed objects is proposed. In terms of measurement noise estimation, accuracy, tracking ability, and numerical stability, we improve the standard UKF . Based on the standard UKF, a wavelet transformation based filter is proposed to estimate the variance of measurement noise. A strong tracking UKF is utilized to improve the tracking ability of catastrophe parameters and states as well as the robustness against inaccurate process noise. A square root argument UKF framework is utilized to improve the accuracy and numerical stability. Monte Carlo simulations and practical application in estimation of the supersonic artillery shell demonstrate that the proposed method can estimate the mutation parameters exactly. The AUKF is better than the general strong tracking Kalman filter and the standard UKF.

\section{References}

[1] L. Ljung. Asymptotic behaviour of the extended Kalman filter as a parameter. IEEE Trans. on Automatic Control, 1979, 24(1): 36-50.

[2] T. Yoshimura, K. Konishi, T. Soeda. A modified extended Kalman filter for linear discrete-time systems with unknown parameters. Automatica, 1981, 17(4): 657-660.

[3] D. H. Zhou, P. M. Frank. Strong tracking filtering of nonlinear time-varying stochastic systems with coloured noise: application to parameter estimation and empirical robustness analysis. International Journal of Control, 1996, 65(2): 295-307.

[4] M. Boutayeb, D. Aubry. A strong tracking extended Kalman observer for nonlinear discrete-time systems. IEEE Trans. on Automatic Control, 1999, 44(8): 1550-1556.

[5] D. J. Jwo, S. H. Wang. Adaptive fuzzy strong tracking extended Kalman filtering for GPS navigation. IEEE Sensors Journal, 2007, 7(5): 778-789.

[6] A. Gelb. Applied optimal estimation. Cambridge: Massachusetts Institute of Technology Press, 1974.

[7] N. J. Gordon, D. J. Salmond, A. F. Smith. Novel approach to nonlinear/non-Gaussian Bayesian state estimation. IEE Proceedings F-Radar and Signal Processing, 1993, 140(2): 107113.

[8] M. S. Arulampalam, S. Maskell, N. Gordon, et al. A tutorial on particle filters for online nonlinear/non-Gaussian Bayesian tracking. IEEE Trans. on Signal Processing, 2002, 50(2): 174188.

[9] S. J. Julier, J. K. Uhlmann, H. F. Durrant-Whyte. A new method for the nonlinear transformation of means and covariances in filters and estimators. IEEE Trans. on Automatic Control, 2000, 45(3): 477-482.

[10] S. J. Julier, J. K. Uhlmann. Unscented filtering and nonlinear estimation. Proceedings of the IEEE, 2004, 92(3): 401-422.

[11] K. Xiong, C. L. Wei, L. D. Liu. Robust unscented Kalman filtering for nonlinear uncertain systems. Asian Journal of Control, 2010, 12(3): 426-433.

[12] J. Ravikumar, S. Subramanian, J. Prakash. Non-linear state estimation using derivative-free filters for a three-phase induction motor model. Australian Journal of Electrical and Electronics Engineering, 2011, 8(3): 231-245.

[13] S. Sarkka. On unscented Kalman filtering for state estimation of continuous-time nonlinear systems. IEEE Trans. on Automatic Control, 2007, 52(9): 1631-1641.

[14] G. Valverde, V. Terzija. Unscented Kalman filter for power system dynamic state estimation. IET Generation, Transmission and Distribution, 2011, 5(1): 29-37.

[15] C. J. Noel, D. Ramdhane. Unscented Kalman filter based nonlinear model predictive control of a LDPE autoclave reactor. Journal of Process Control, 2011, 21(9): 1332-1344.

[16] V. Chari, C. V. Jawahar. Multiple plane tracking using unscented Kalman filter. Proc. of the IEEE/Robotics Society of Japan International Conference on International Robotics and Systems, 2010: 2914-2919.

[17] C. Liu, P. Shui, S. Li. Unscented extended Kalman filter for target tracking. Journal of Systems Engineering and Electronics, 2011, 22(2): 188-192.

[18] D. J. Jwo, C. N. Lai. Unscented Kalman filter with nonlinear dynamic process modeling for GPS navigation. GPS Solution, 2008, 12(4): 249-260.

[19] J. Liu , J. Ma, J. W. Tian. Pulsar/CNS integrated navigation based on federated UKF. Journal of Systems Engineering and Electronics, 2010, 21(4): 675-681.

[20] C. Kim, R. Sakthivel, W. K. Chung. Unscented fast SLAM: a robust and efficient solution to the SLAM problem. IEEE Trans. on Robotics, 2008, 24(4): 808-820.

[21] L. Seongsoo, L. Sukhan, K. Dongsung. Recursive unscented Kalman filtering based SLAM using a large number of noisy observations. International Journal of Control, Automation, and Systems, 2006, 4(6): 736-747.

[22] K. Xiong, C. W. Chan, H. Y. Zhang. Detection of satellite attitude sensor faults using the UKF. IEEE Aerospace and Electronic Systems Magazine, 2007, 43(2): 480-491. 
Fang Deng et al.: Adaptive unscented Kalman filter for parameter and state estimation of nonlinear high-speed objects

[23] S. J. Julier, J. K. Uhlmann. Reduced sigma point filters for the propagation of means and covariances through nonlinear transformations. Proc. of American Control Conference, 2002: 887-892.

[24] M. R. Van, E. A. Wan. The square-root unscented Kalman filter for state and parameter-estimation. Proc. of the IEEE International Conference Acoustics Speech Signal Processing, 2001: 3461-3464.

[25] J. S. Kim, D. R. Shin, E. Serpedin. Adaptive multiuser receiver with joint channel and time delay estimation of CDMA signals based on the square-root unscented filter. Digital Signal Processing, 2009, 19(3): 504-520.

[26] D. H. Zhou, Y. G. Xi. Modem fault diagnosis and fault tolerant control. Beijing: Tsinghua University Press, 2000: 96-97. (in Chinese)

[27] R. Fitzgerald. Divergence of the Kalman filter. IEEE Trans. on Automatic Control, 1971, 16(6): 736-747.

[28] A. P. Sage, G. W. Husa. Adaptive filtering with unknown prior statistics. Proc. of Joint Automatic Control Conference, 1969: 760-769.

[29] C.Y. Zhang. Approach to adaptive filtering algorithm. Acta Aeronautica et Astronautica Sinica, 1998, 19(7): 96-99. (in Chinese)

[30] R. G. Reynolds. Robust estimation of covariance matrices. IEEE Trans. on Automatic Control, 1990, 35(9): 10471051.

[31] Z. M. Durovic, B. D. Kovacevic. Robust estimation with unknown noise statistics. IEEE Trans. on Automatic Control, 1998, 44(6): 1292-1296.

[32] E. Cinquemani, G. Pillonetto. Wavelet estimation by Bayesian thresholding and model selection. Automatica, 2008, 44(9): 2288-2297.

[33] R. Xia, K. Meng, F. Qian, et al. Online wavelet denoising via a moving window. Acta Automation Sinica, 2007, 33(9): 897901. (in Chinese)

[34] S. Mallat. A wavelet tour of signal processing. 2nd ed. Beijing: China Machine Press, 2003. (in Chinese)

[35] L. Xu, J. Zhang, Y. Yan. A wavelet-based multisensor data fusion algorithm. IEEE Instrumentation and Measurement Magazine, 2004, 53(6): 1539-1545.

[36] S. Godbole. Kalman filtering with no a priori information about noise-white noise case: identification of covariances. IEEE Trans. on Automatic Control, 1974, 19(5): 561-563.

[37] D. L. Donoho, I. M. Johnstone. Ideal spatial adaptation via wavelet shrinkage. Biometrika, 1994, 81(3): 425-455.

[38] D. L. Donoho, I. M. Johnstone. Adapting to unknown smoothness via wavelet shrinkage. Journal of the American Statistical Association, 1995, 90(432): 1200-1224.

[39] Y. Gao, J. Q. Zhang. Kalman filter with wavelet based unknown measurement noise estimation and its application for information fusion. Acta Electronic Sinica, 2007, 35(1): 108111. (in Chinese)

[40] R. Van der Merwe. Sigma-point Kalman filters for probabilistic inference in dynamic state-space models. Stellenbosch: University of Stellenbosch, 2004.

[41] S. J. Julier. The spherical simplex unscented transformation. Proc. of the American Control Conference, 2003: 24302434.

[42] K. Xiong, H. Y. Zhang, C. W. Chan. Performance evaluation of UKF-based nonlinear filtering. Automatica, 2006, 42(2): 261-270.

[43] J. Chen, F. Deng, W. J. Chen. Parameters identification from indirect data and its application in the identification of ballistic parameters. Journal of Beijing Institute of Technology, 2007, 27(1): 118-122. (in Chinese)

[44] Z. G. Yan, Z. K. Qi. Firing table technologies. Beijing: National Defense Industry Press, 2000.

\section{Biographies}

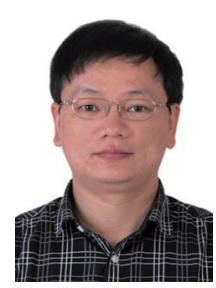

Fang Deng was born in 1981. He received his B.E. degree and Ph.D. degree in control science and engineering from Beijing Institute of Technology, Beijing, China, in 2004 and 2009, respectively. He is currently a lecturer and a master supervisor with the School of Automation, Beijing Institute of Technology. His current research interests include nonlinear estimation, fault diagnosis and fire control

systems.

E-mail: dengfang@bit.edu.cn

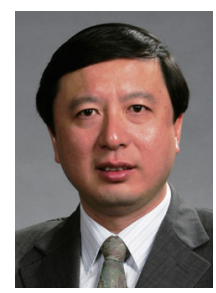

Jie Chen was born in 1965. He received his B.E. degree, M.E. degree and Ph.D. degree in control science and engineering from Beijing Institute of Technology, Beijing, China, in 1986, 1996 and 2000 , respectively. He is currently a professor and a Ph.D. supervisor with the School of Automation, Beijing Institute of Technology. His main research interests include multi-index optimization, multiobjective decision and control, intelligent control, and constrained nonlinear control.

E-mail: chenjie@bit.edu.cn

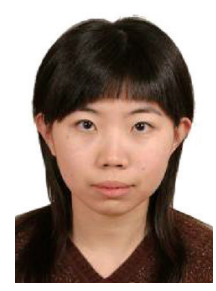

Chen Chen was born in 1982. She received her B.E. degree and $\mathrm{Ph} . \mathrm{D}$. degree in control science and engineering from Beijing Institute of Technology, Beijing, China, in 2004 and 2009, respectively. She is a lecturer at Beijing Institute of Technology. Her research interest covers complicated system multiobject optimization and distributed simulation. Email: xiaofan@bit.edu.cn 